\title{
ALGUNS ASPECTOS DA MORTALIDADE DE CRIANÇAS EM OSASCO, S.P., BRASIL *
}

Cyro CIARI Jr.

Arnaldo Augusto Franco de SIQUEIRA

Pedro Augusto Marcondes de ALMEIDA

Alfredo ARNONI

RSPU-B/172

CIARI Jr., C. et al. - Alguns aspectos da mortalidade de crianças em Osasco, S.P., Brasil. Rev. Saúde públ., S. Paulo, 7: 199-206, 1973.

Resumo: Foi estudada a mortalidade de menores de um ano do municipio de Osasco, no periodo de 1967 a 1971, utilizando-se a mortalidade proporcional por idade, bem como os coeficientes de mortalidade infantil $e$ coeficientes especificos de mortalidade para menores de um dia, crianças de um a 6 dias e de 7 a 27 dias. Com os resultados obtidos pretende-se caracterizar o municipio de Osasco como uma área não desenvolvida, quanto a este aspecto na qual está ocorrendo uma piora das condições de saúde, da assistência à infância e à maternidade, bem como do sanexmento do meio.

Unitermos: Mortalidade neonatal *; Mortalidade infantil *; Assistência pré-natal *; Saúde materna *

Já tem sido demonstrada a falta de credibilidade dos dados de mortalidade disponíveis entre nós. Assim é que, na quase totalidade dos casos, os atestados de óbito, fonte primordial de informações, são preenchidos erradamente, o que quase impede o seu estudo. Esses erros dificultam especialmente a determinação da causa básica do óbito. Informações auxiliares, como a idade do indivíduo ao morrer, são quase sempre certas 4 .

Apesar do estudo dos óbitos segundo a idade ao morrer não trazer tantas informações como o estudo da mortalidade por causas, pode dar-nos subsídios para determinar níveis de saúde de uma área, bem como caracterizar deficiências das atividades de saúde.

A maior fidedignidade desses dados em relação aos de causas de b́bitos justifica o seu estudo.

Neste trabalho foram analisados, segundo a idade, os óbitos de menores de um ano registrados no município de Osasco no periodo de $1 . \% / 1 / 1967$ a 31/12/1971.

* Da Disciplina de Higiene Materna do Departamento de Prática de Saúde Pública da Faculdade de Saúde Pública da USP - Av. Dr. Arnaldo, 715 - São Paulo, S.P. - Brasil. 
CIARI Jr., C. et al. - Alguns aspectos da mortalidade de crianças em Osasco, S.P., Brasil. Rev. Saúde públ., S. Paulo, 7: 199-206, 1973.

Do estudo das proporções dos óbitos de menores de um dia, crianças de um a 6 dias e de 7 a 27 dias na mortalidade neonatal, e desses b́bitos todos em relação ao restante da mortalidade infantil, pretende-se classificar o município de Osasco como área em desenvolvimento neste aspecto e na qual vem ocorrendo uma piora das condições de saúde, com prováveis reflexos no desenvolvimento social global e na assistência à infância e à maternidade.

\section{MATHRIAL E METODOS}

Foram selecionados para estudo os obitos de menores de um ano registrados no município de Osasco no período de 1. $\% / 1 / 1967$ a $31 / 12 / 1971$.

Esses óbitos foram, ano a ano, distribuidos em dois grupos. O primeiro abrangeu crianças falecidas com menos de 28 dias de idade e o segundo, crianças falecidas com 28 dias a um ano.

O grupo de menores de 28 dias foi ainda subdivido em menores de um dia, crianças de um a 6 dias e crianças de 7 a 27 dias.
A seguir foram calculadas as frequiências relativas de cada grupo, bem como a proporção entre os óbitos de menores de 28 dias (óbitos neonatais) e óbitos de crianças de 28 dias a um ano (6bitos pós-neonatais - período infantil tardio).

Os resultados obtidos foram comparados com os da Investigação Interamericana de Mortalidade na Infância, relativos à cidade de São Paulo ${ }^{2}$.

Foram escolhidos os resultados dessa pesquisa para comparação, em virtude de serem eles virtualmente exatos no que diz respeito à idade ao morrer, uma vez que a idade da criança falecida era determinada através de entrevistas com a familia da criança e consulta a arquivos hospitalares.

Finalmente, a partir de dados fornecidos pelo Cartório de Registro Civil de Osasco, foram calculados os respectivos coeficientes de mortalidade.

\section{RESULTADOS}

Os óbitos neo-natais do município de Osasco, segundo idade ao morrer, constituem a Tabela 1.

T A B E L A 1

Obltos neonatais registrados no municipio de Osasco no perfodo de $1.0 / 1 / 1967$ a 31/12/1971, segundo a Idade ao morrer.

\begin{tabular}{|c|c|c|c|c|c|c|c|c|c|c|}
\hline \multirow[t]{2}{*}{ Grupo etário } & \multicolumn{2}{|c|}{1967} & \multicolumn{2}{|c|}{1968} & \multicolumn{2}{|c|}{1969} & \multicolumn{2}{|c|}{1970} & \multicolumn{2}{|c|}{1971} \\
\hline & n.o & $\%$ & n. ${ }^{\circ}$ & $\%$ & n. ${ }^{\circ}$ & $\%$ & n.o & $\%$ & n..$^{\circ}$ & $\%$ \\
\hline Menores de um dia & 42 & 23,2 & 41 & 19,4 & 39 & 15,7 & 38 & 13,3 & 76 & 17,3 \\
\hline Um a 6 dias & 91 & 50,3 & 68 & 32,2 & 97 & 39,1 & 80 & 27,8 & 110 & 25,5 \\
\hline 7 a 27 dias & 48 & 26,5 & 102 & 48,4 & 112 & 45,2 & 169 & 58,9 & 243 & 56,9 \\
\hline TOTAL & 181 & 100,0 & 211 & 100,0 & 248 & 100,0 & 287 & 100,0 & 432 & 100,0 \\
\hline
\end{tabular}


CIARI Jr., C. et al. - Alguns aspectos da mortalidade de crianças em Osasco, S.P., Brasil. Rev. Saúde públ., S. Paulo, 7: 199-206, 1973.

Do exame dessa Tabela pode-se verificar a existência de duas tendências contrárias: de um lado, uma diminuição da proporção de óbitos de menores de um dia e de crianças de um a 6 dias; de outro, um nítido aumento relativo aos óbitos de crianças de 7 a 27 dias.

Em números absolutos houve grande aumento dos óbitos neonatais que, de $181 \mathrm{em} \mathrm{1967,} \mathrm{atingiram} \mathrm{a} 432 \mathrm{em} \mathrm{1971,}$ mais do dobro, portanto. A Figura 1 ilustra o fenômeno.

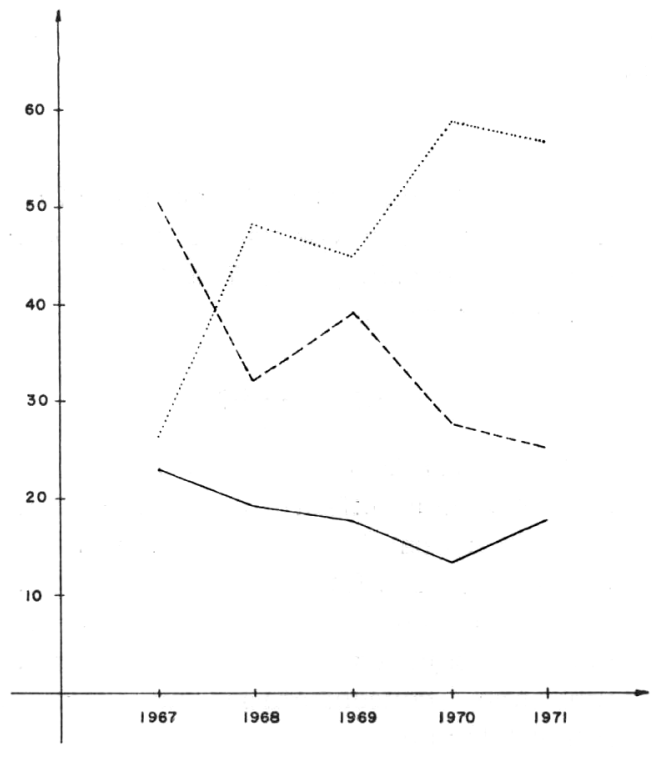

\section{LEGENDA}

- OBITOS DE MENORES DE I DIA

--- OBITOS DE CRIANÇAS DE IAG DIAS

OBI........ OBIOS DE CRIANCGAS DE TA 27 DIAS

Fig. 1 - Proporções dos óbitos neonatais segundo a idade ao morrer.

Município de Osasco.

Perfodo de $1 / 1 / 67$ a $31 / 12 / 71$.

Quanto à importância dos componentes neonatal e infantil tardio na mortalidade infantil, os óbitos neonatais fo- ram, ano a ano, responsáveis por cerca de $40 \%$ do total de óbitos de menores de um ano; esses valores estão na Tabela 2 .

Quanto aos coeficientes de mortalidade neonatal e mortalidade infantil, nota-se um aumento constante e expressivo, desde 1967 até 1971 (Figuras 1 e 2).

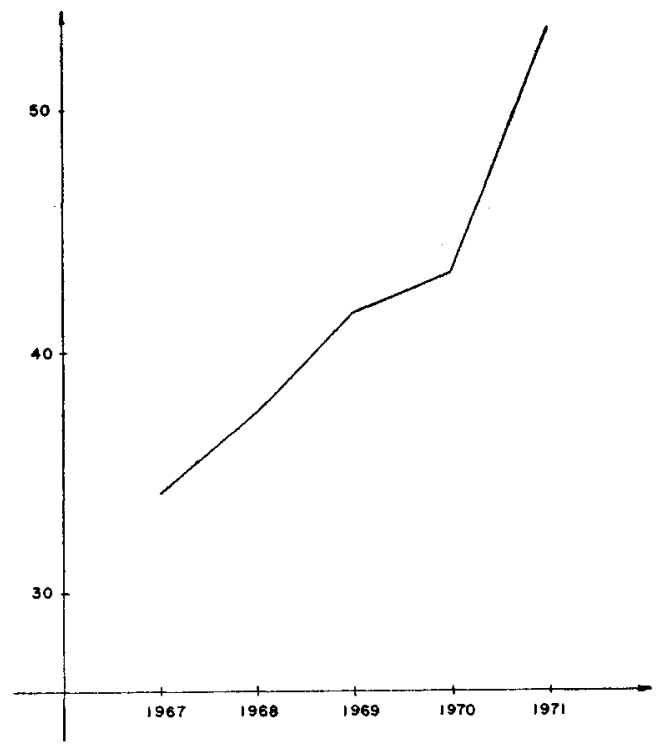

Fig. 2 - Mortalidade neonatal. Municipio de Osasco. Período de 1967 a 1971.

O coeficiente de mortalidade neonatal de Osasco, em 1967, era de 34,1 por mil nascidos vivos. Esse valor foi aumentando ano a ano e em 1971 atingiu a cifra de 53,4 - óbitos de menores de 28 dias por mil nascidos vivos.

A mortalidade infantil, que em 1967 situava-se em $83,5 \%$ também elevou-se e atingiu o valor de 129,6 óbitos por mil nascidos vivos em 1971.

Subdividindo o período neonatal em menores de um dia, um a 6 dias e 7 a 27 dias, podemos construir coeficientes específicos de mortalidade por idade, relacionando os óbitos verificados nesses 
CIARI Jr., C. et al. - Alguns aspectos da mortalidade de crianças em Osasco, S.P., Brasil. Rev. Saúde públ., S. Paulo, 7: 199-206, 1973.

\section{T A B E L A 2}

Proporçāo dos componentes neonatal e infantil tärdio na mortalidade de menores de um ano, em Osasco, no período de $1 . \% / 1 / 67$ a $31 / 12 / 71$.

\begin{tabular}{|c|c|c|c|c|c|c|c|c|c|c|}
\hline \multirow{2}{*}{ Componente } & \multicolumn{2}{|c|}{1967} & \multicolumn{2}{|c|}{1968} & \multicolumn{2}{|c|}{1969} & \multicolumn{2}{|c|}{1970} & \multicolumn{2}{|c|}{1971} \\
\hline & n. ${ }^{\circ}$ & $\%$ & n. ${ }^{\circ}$ & $\%$ & n. ${ }^{\circ}$ & $\%$ & n. ${ }^{\circ}$ & $\%$ & n. ${ }^{\circ}$ & $\%$ \\
\hline Neo-natal & 181 & 40,9 & 211 & 38,0 & 248 & 42,0 & 287 & 43,3 & 432 & 41,2 \\
\hline Infantil tardio & 262 & 59,1 & 344 & 62,0 & 342 & 58,0 & 376 & 56,7 & 616 & 58,8 \\
\hline TOTAL & 443 & 100,0 & 590 & 100,0 & 663 & 100,0 & 663 & 100,0 & 1048 & 100,0 \\
\hline
\end{tabular}

Fonte: Departamento Técnico Normativo da Secretaria da Saúde de Osasco.

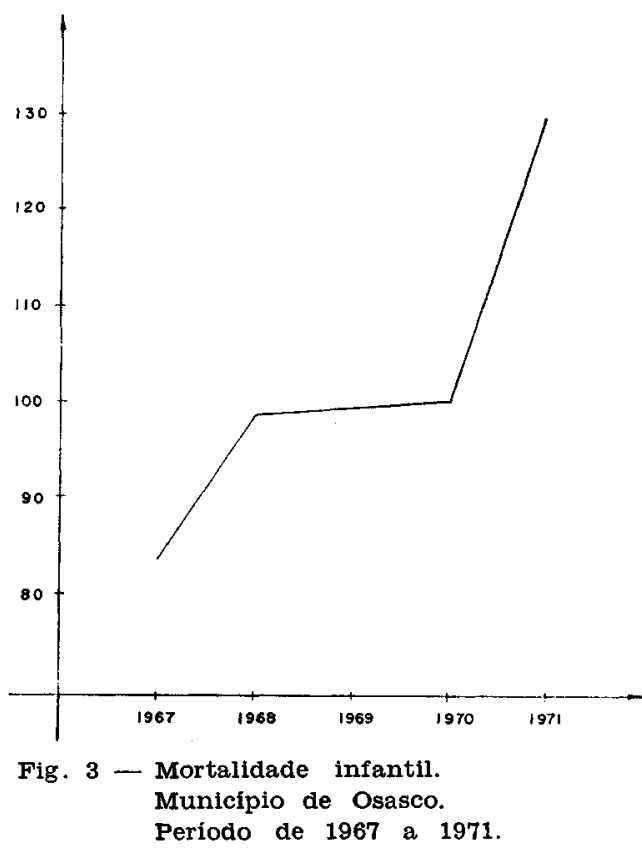

grupos etários ao total de nascidos vivos. fi claro que, assim procedendo, a soma dos coeficientes assim obtidos deve ser igual ao coeficiente de mortalidade neonatal.

Assim, houve flutuações nos coeficientes para menores de um dia e crianças de um a 6 dias, ao lado de um nítido aumento para 0 coeficiente relativo às crianças falecidas com 7 a 27 dias de idade (Figura 4).

\section{DISCUSSAO}

Tem-se verificado que em áreas onde a mortalidade infantil é baixa, o componente neonatal aparece em maior proporção que o infantil tardio. O contrário ocorre em áreas de alta mortalidade, ou seja, os óbitos de menores de um ano concentram-se no período infantil tardio.

Além disso, sempre que ocorre uma redução na mortalidade infantil, ela se dá à custa da diminuição da mortalidade infantil tardia para depois diminuir a mortalidade neonatal. Em outras palavras: é mais fácil conseguir-se a redução da mortalidade pos-neonatal.

A mortalidade neonatal no município de São Paulo representa $\mathbf{5 0} \%$ da infantil. Esse achado, que à primeira vista poderia indicar uma tendência de comportamento semelhante ao de regiōes desenvolvidas, deve-se ao fato de ser a 
CIARI Jr., C. et al. - Alguns aspectos da mortalidade de crianças em Osasco, S.P., Brasil. Rev. Saúde públ., S. Paulo, 7: 199-206, 1973.

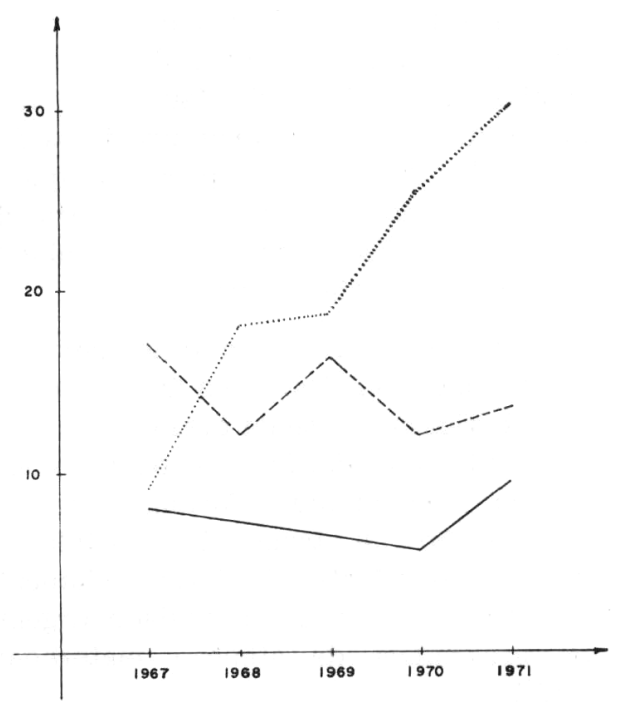

$$
\begin{aligned}
& \text { 1 } 24 \mathrm{~h} . \\
& \ldots \text { IA } 6 \text { DIAS } \\
& \text { TA } 27 \text { DIAS }
\end{aligned}
$$

Fig. 4 - Coeficientes especificos de mortalidade segundo a idade. Município de Osasco.

Período de 1967 a 1971.

mortalidade neonantal tão elevada que atinge $50 \%$ de uma mortalidade infantil também elevada ${ }^{3}$.

Em Osasco, como nas regiōes subdesenvolvidas, o componente neonatal da mortalidade infantil variou em torno de $40 \%$, o que sugere que seja baixo o nível de saúde e desenvolvimento social da área, bem como precárias as atividades de assistência materno-infantil.

Analisando a Tabela 1, a impressão inicial é a de que a assistência ao parto esteja melhorando, isto é, cada vez menor proporção de crianças falecem no primeiro dia de vida (período em que o atendimento obstétrico exerce grande influência na mortalidade). Em números absolutos, no entanto, houve um aumento tanto dos óbitos de menores de um dia quanto das crianças de um a 6 dias. Essa menor proporção de óbitos na primeira semana de vida corre por conta, pois, de um espetacular incremento dos obitos de crianças de 7 a 27 dias.

Em áreas desenvolvidas a grande maioria dos óbitos neonatais concentra-se no primeiro dia de vida. Um certo número de crianças sobrevive às primeiras 24 horas e vem a falecer antes de completar 7 dias de vida. Finalmente, uma pequena parcela que raramente alcança os $10 \%$ dos óbitos neonatais falece entre 7 e 28 dias de idade 1.

$$
\text { T A B E L A } 3
$$

obitos neonatais segundo a idade ao morrer ocorridos no município de São Paulo, no período de $1 / 6 / 68$ a $31 / 5 / 70$.

\begin{tabular}{l|c|c}
\hline Grupo etário & N.o & $\%$ \\
\hline $\begin{array}{l}\text { menores de um } \\
\text { dia }\end{array}$ & 2662 & 32,3 \\
um a 6 dias & 2325 & 28,1 \\
7 a 27 dias & 3277 & 39,6 \\
\hline TOTAL & 8264 & 100,0
\end{tabular}

Fonte: Investigaçáo Interamericana de Mortalidade na Infâncla.

Se em São Paulo, de acordo com a Tabela 3, já ocorre um grande número de óbitos neonatais tardios ( 7 a 27 dias), em Osasco, que apresentava uma distribuição semelhante em 1967, vem ocorrendo um predomínio sempre maior dos óbitos nesse grupo etário, de tal forma que em 1970 e 1971 quase $60 \%$ dos obitos neonatais verificaram-se nesse grupo.

Esse fenômeno sugere, assim, que o nível de saúde, o desenvolvimento social, assistência materno-infantil, o equi- 
CIARI Jr., C. et al. - Alguns aspectos da mortalidade de crianças em Osasco, S.P., Brasil. Rev. Saúde públ., S. Paulo, 7: 199-206, 1973.

pamento hospitalar (berçários) da área, que já eram deficientes em 1967, estejam piorando inexoravelmente.

E preciso que se diga, no entanto, que não se pode afirmar categoricamente essas hipóteses, pois não se conhece o comportamento de uma variável de importância fundamental no estudo da mortalidade de uma área, ou seja, o problema da evasão de óbitos para outras áreas (no caso, São Paulo), bem como da invasão de casos provenientes de áreas vizinhas.

A experiência na área demonstra que - número de crianças residentes em Osasco e que eram levadas para São Paulo, para tratamento, onde faleciam, era enorme. Com a ampliação da rede hospitalar e com a melhora das condições de atendimento de Osasco, é possível que um grande número de crianças tenham passado a ser atendidas no local, com o imediato reflexo no aumento do número de óbitos.

Parece-nos, no entanto, que o aumento da mortalidade proporcional de crianças de 7 a 27 dias dificilmente poderia ser explicado apenas em razão da diminuição da evasão de óbitos.

Essa piora, aliás, não é de estranhar, uma vez que vem ocorrendo no município de São Paulo ${ }^{3}$ e, provavelmente, em toda a Grande São Paulo.

Comparando os coeficientes de mortalidade obtidos em Osasco com os correspondentes de São Paulo, verifica-se que em 1967 a mortalidade neonatal de Osasco $(34,1 \%$ n.v. $)$ era semelhante à de São Paulo em 1968/70 (34,5\% n.v.).

$O$ coeficiente de mortalidade neonatal foi aumentando e, em 1971, atingiu a cifra de $53,4 \%$ n.v. A mortalidade infantil atingiu, em 1971, o valor de $129,6 \%$ n.v. Esses valores são altamente insatisfatórios.
Se a evasão de óbitos não permite conhecer os volores exatos desses coeficientes no periodo de 1967 a 1971, é óbvio que os valores mais próximos dos reais são os de 1971, época em que a evasão diminuiu pela maior oferta de leitos à população, seja de maternidade (e berçário), seja de pediatria.

Assim, é possível que o aumento da mortalidade verificado em Osasco, desde que corrigido à base da evasão de óbitos, não tenha sido tão impressionante como parece. De qualquer forma, a simples constatação de taxas de mortalidade neonatal $e$ infantil tão inesperadamente elevadas indicam a necessidade urgente de programas de atenção materno-infantil.

Analisando-se, finalmente, os coeficientes específicos de mortalidade em cada grupo do período neonatal, pode-se dizer que, apesar de tanto os óbitos de menores de um dia quanto os de crianças de um a 6 dias terem se tornado proporcionalmente menos representativos, os coeficientes para esses grupos etários apresentam valores muito elevados. $O$ coeficiente específico para menores de um dia variou de $7,8 \%$ n.v. em 1967 a $9,2 \%$ n.v. em 1971; para as crianças falecidas no grupo um a 6 dias, esses valores chegam a, respectivamente, $17,1 \%$ n.v. e $13,6 \%$ n.v. (Figura 4). Apenas a título de comparação, se tomarmos o caso da Holanda, a mortalidade infantil no país como um todo não atinge a 12 óbitos por mil nascidos vivos 1 .

Se a mortalidade para os grupos de menores de um dia e crianças de um a 6 dias está flutuando, apesar de em valores elevadíssimos, o mesmo não ocorreu para o grupo de 7 a 27 dias, em que se verificou um grande aumento, de 9 por mil nascidos vivos em 1967, para 30 por mil nascidos vivos em 1971. Fica, assim, bem amparada a hipótese de estar havendo uma piora das condições de 
CIARI Jr., C. et al. - Alguns aspectos da mortalidade de crlanças em Osasco, S.P., Brasil. Rev. Saúde públ., S. Paulo, 7: 199-206, 1973.

saúde, desenvolvimento social global e da assistência à infância e à maternidade do município de Osasco, piora essa tornada mais importante em virtude de ter ocorrido a partir de condições iniciais já bastante deficientes.

Não é difícil procurar determinar as principais causas desse fenômeno observado em Osasco.

Se na mortalidade de menores de um dia o atendimento obstétrico e o prénatal são de grande importância, no grupo de um a 6 dias aparece um terceiro fator de aumento de mortalidade, que é a precariedade do atendimento ao recém-nascido, com berçários mal aparelhados, falta de pessoal especializado, inexistência de berçários de prematuros e, pela premência de vagas, ou melhor, necessidade de maior rotatividade dos leitos, com altas hospitalares muito precoces.

O grande número de óbitos de crianças de 7 a 27 dias, além dos fatores já citados e cuja ação ainda se faz sentir nessa idade, decorre particularmente de condições ambientais (saneamento do meio, serviços de assistência à criança e de prevenção de doenças), não se podenđo deixar de mencionar o papel de difusores de doenças (diarréia) que os hospitais desempenham no drama da mortalidade.

\section{CONCLUSOES}

1) O elevado coefiente de mortalidade de menores de um dia indica a necessidade de melhoria não só da assistência ao parto como de consultas de pré-natal.

2) O número significativo de óbitos de crianças de um a 6 dias indica a necessidade de melhoria dos equipamentos de assistência ao recém nascido, bem como a revisão dos conceitos de alta precoce, e também de um sistema para $o$ atendimento específico de prematuros.

3) A elevada proporção de óbitos de crianças de 7 a 27 dias indica a necessidade de melhoria de condições de vida (Saneamento do meio), bem como de serviços de assistência à criança e rrevenção de doenças.

RSPU-B/172

CIARI Jr., C. et al. - [Some aspects of infant mortality. in Osasco, S. Paulo, Brazil]. Rev. Saúde públ., S. Paulo, 7: 199-206, 1973.

Summary: The infant mortality was studied in Osasco, S.P. (Brazil) using the proportional mortality per age, as well as the coefficient of neonatal mortality, infant mortality and specific coefficient of mortality of less than one day old children, children from one to 6 days old and from 7 to 27 days old. With the obtained results it is intended to characterize Osasco in some aspects as a non developed area, where not only the health conditions and infant maternal care but also environment sanitation are getting worse.

UNITERMS: Neonatal mortality *; Infant mortality*; Pre-natal care*; Maternal health *. 
CIARI Jr., C. et al. - Alguns aspectos da mortalidade de crlanças em Osasco, S.P., Brasil. Rev. Saude puibl., S. Paulo, 7: 199-206, 1973.

\section{REFERENCIAS BIBLIOGRAFICAS}

1. INTERNACIONAL Comparison of Perinatal and Infant Mortality: The United States and Six West European Countries. Vital Hlth. Stat., Series 3, (6) :1-97, 1967.

2. LAURENTI, R. A. - Investigação Interamericana de Mortaltdade na Infância em São Paulo, Brasil. Rev. Saúde públ., S. Paulo 3:225-9, 1969.
3. LAURENTI, R. \& SIQUEIRA, A. A. F. O problema da mortalidade neonatal em São Paulo, Brasil. Rev. Saúde públ., S. Paulo, 6:45-55, 1972.

4. MILANESE, M. L. \& LAURENTI, R. O estudo interamericano de mortalidade em Sáo Paulo: I - Estado atual da certificação médica de causa de óbitos no Distrito da Capital. Rev. Ass. med. bras., 10:111-6, 1964.

Recebido para publicação em 11-5-1973

Aprovado para publicação em 10-7-1973 